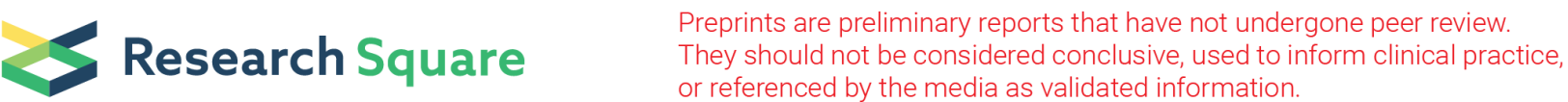

\section{Mental Health Problems Are the Leading Cause of Hospitalization Among Adolescents Accessing a Pediatric Emergency Department in Italy.}

Giorgio Cozzi ( $\sim$ giorgio.cozzi@burlo.trieste.it)

1) Institute for Maternal and Child Health IRCCS Burlo Garofolo

\section{Lisa Passaglia}

University of Trieste Clinical Department of Biomedicine: Universita degli Studi di Trieste Dipartimento Universitario Clinico di Scienze Mediche e Chirurgiche e della Salute

\section{Anna Agrusti}

University of Trieste Clinical Department of Biomedicine: Universita degli Studi di Trieste Dipartimento Universitario Clinico di Scienze Mediche e Chirurgiche e della Salute

\section{Manuela Giangreco}

Institute for Maternal and Child Health: IRCCS materno infantile Burlo Garofolo

\section{Rita Giorgi}

Institute for Maternal and Child Health: IRCCS materno infantile Burlo Garofolo

\section{Ingrid Rabach}

Institute for Maternal and Child Health: IRCCS materno infantile Burlo Garofolo

\section{Claudio Germani}

Institute for Maternal and Child Health: IRCCS materno infantile Burlo Garofolo

\section{Egidio Barbi}

University of Trieste Clinical Department of Biomedicine: Universita degli Studi di Trieste Dipartimento Universitario Clinico di Scienze Mediche e Chirurgiche e della Salute

\section{Research}

Keywords: pediatric emergency department, adolescence, mental health

Posted Date: December 1st, 2020

DOI: https://doi.org/10.21203/rs.3.rs-114477/v1

License: (c) (i) This work is licensed under a Creative Commons Attribution 4.0 International License. Read Full License 


\section{Abstract}

Aim

Describe the use of the pediatric emergency department of a tertiary-level children's hospital in Italy by adolescents.

\section{Methods}

This retrospective study was based on the medical records of patients aged 13 to 17 years, who accessed the pediatric emergency department of the Institute for Maternal and Child Health IRCCS Burlo Garofolo, from 1 January to 31 December 2018. For each patient, we reported: age, gender, triage category, chief complaint, diagnosis, admission status, and hospital ward of destination. The primary outcome was to describe the use of the department in terms of leading causes of access, diagnoses, and outcomes.

Results

During the study period, 3062 adolescents accessed to the department, for a total of 3895 unscheduled visits. The principal causes of access were trauma (45.3\%) and organic diseases (38.8\%). 249 adolescents (6.4\%) had mental health problems.

One-hundred-forty-two adolescents (3.6\%) were admitted to the hospital, 54 of whom to the neuropsychiatric ward, for mental health problems. Compared to younger patients entering the department, adolescents were admitted to the neuropsychiatric ward more frequently $(p<0.001)$.

\section{Conclusions}

In this series, mental health problems were a frequent cause of urgent access to the hospital and the leading cause of urgent hospitalization among adolescents.

\section{Background}

Adolescence represents a particular moment in life, characterized by the need to experiment and discover crucial for the construction of each person's identity. In a simplified view, the biological basis of adolescence neurocognitive development is underpinned by the maturation of the prefrontal cortex, the region of the brain regulating the rational control of the events. During adolescence, the experience induces mechanisms of pruning and remodeling neuronal connections so that the cortical plasticity typical of this age makes adolescents more vulnerable to the social demands. Overall, the interaction between these mechanisms and the pressure of the society can have substantial psychological consequences. $(1,2)$ Adolescents experience an increase in risk behaviors, associated with a higher incidence of major trauma and delayed access to medical services. (3-5) Concern about adolescent mental health has grown in recent years: current studies report a fivefold increase in students' mental health disorders, particularly among young women, with addiction to social media "judgement," which 
plays a leading role in focus on psychological stress and social isolation. (6) Few studies have been published on adolescent use of emergency services $(7,8)$ in contrast to the growing concern for this age group's well-being. (1)

This study aimed to describe the use of the pediatric emergency department (PED) of a children's hospital by adolescents.

\section{Methods}

This retrospective study involved patients evaluated at the PED of the Institute for Maternal and Child Health IRCCS Burlo Garofolo (Trieste, Italy), a tertiary level, University teaching, children's hospital. Trieste is a city of 200.000 inhabitants located in the North East of Italy. Our PED is the only one in town, and has an annual census of 25.000 patients, from zero to 17 years of age.

The study protocol was approved by the Institutional Review Board of the Institute (RC26/2020).

We collected data from the electronic database of the PED, considering the medical records of patients aged 13 to 17 years of age, from 1 January to 31 December 2018. We excluded the records regarding a scheduled re-visit. For each patient, we reported age, gender, date of the visit, nursing triage category, patient's chief complaint, discharge diagnosis, admission status, and hospital ward of destination.

Pediatric nurses, with at least five years of experience, performed the triage using the Italian National Triage Category system, which consists of four priority levels with increasing severity: white (not-urgent), green (minor emergencies), yellow (urgent) and red (emergency/resuscitation).

We classified the diagnosis into five main domains: trauma, organic diseases, functional diseases, mental health problems, and others. Functional diseases included conditions which fulfilled specific criteria for a defined functional syndrome $(9,10)$ or patients whose medical history, clinical examination, test results and response to pharmacological therapies were not consistent with either organic, traumatic or psychiatric condition.

We compared data about triage codes, rate of hospitalization and ward of destination of adolescents with data collected from the population of younger patients seen in our PED in the same period of time.

The study's primary outcome was to describe the use of the PED by adolescents in terms of causes of access, diagnosis and outcomes.

The secondary endpoint was to compare the number of admissions and the hospital ward of destination of adolescents compared to the younger patients accessing the PED.

\section{Statistical analysis.}


The continuous variables were described as median and interquartile ranges, while absolute frequency and percentage were used to describe categorical variables. The Fisher Exact test or the Chi-square test were employed to verify the associations between categorical variables. A p-value $<0.05$ was considered significant.

\section{Results}

From 1 January to 31 December 2018, 24.599 visits were made to the PED, 4539 (18.5\%) of which concerned adolescents, aged 13 to 17 . We excluded 644 accesses for scheduled re-visits, so the total number of accesses among adolescents was 3895. The total amount of adolescents evaluated was 3062,1310 females (42.8\%), and 1752 males (57.2\%), with an average mean age of 15 years old $+/-1.8$.

Table 1 shows the main characteristics of the adolescents accessing to the PED in the study period. 
Table 1

Main characteristics of the adolescents accessing the PED

\begin{tabular}{|ll|}
\hline & $\begin{array}{l}\text { Visits of adolescents at the } \\
\text { PED }\end{array}$ \\
\hline Visits, $\mathbf{n}(\%)$ & $3895(100)$ \\
\hline Patients, $\mathbf{n}$ & 3062 \\
Age in years, mean, (standard deviation) & $15(+/-1.8)$ \\
Female, $\mathbf{n}(\%)$ & $1310(42.8)$ \\
\hline Presenting complaints, $\mathbf{n}(\%)$ & $1362(35)$ \\
- Limb trauma & $270(6.9)$ \\
- Skin rash & $251(6.4)$ \\
- Abdominal pain & $153(3.9)$ \\
- Psycho-motor agitation & $1859(47.7)$ \\
- Other & \\
\hline Diagnosis at discharge, $\mathbf{n}(\%)$ & $1764(45.3)$ \\
- Trauma & $1511(38.8)$ \\
- Organic disease & $249(6.4)$ \\
- Mental health problems & $152(3.9)$ \\
- Functional disease & $219(5.6)$ \\
\hline - Other & $249(6.4)$ \\
\hline Visits related to adolescents with mental health problems, & $158(67)$ \\
\hline Female, $\mathbf{n}$ (\%) & \\
\hline
\end{tabular}




\begin{tabular}{|ll|}
\hline & Visits of adolescents at the \\
\hline $\begin{array}{ll}\text { Symptoms related to adolescents with mental health problems, } \mathbf{n} \\
\text { (\%) }\end{array}$ & $139(55.8)$ \\
- Psychomotor agitation & $24(9.6)$ \\
- Altered state of consciousness & $12(4.8)$ \\
- Headache & $11(4.4)$ \\
- Chest pain & $9(3.6)$ \\
- Dizziness & $5(2.0)$ \\
- Anorexia & $5(2.0)$ \\
- Tachycardia & $5(2.0)$ \\
- Respiratory distress & $5(2.0)$ \\
- Abdominal pain & $34(13.7)$ \\
- Other & \\
\hline Diagnosis related to adolescents with mental health problems, $\mathbf{n}$ & $58(23.5)$ \\
(\%) & $43(17.4)$ \\
- Anxiety & $14(5.8)$ \\
- Psychomotor-agitation & $9(3.3)$ \\
- Behavioral disorder & $6(2.5)$ \\
- Depression & $8(2.9)$ \\
- Suicide attempt & $6(2.2)$ \\
- Psychosis & $105(42)$ \\
- Somatic symptom disorder & \\
- Other & \\
\hline
\end{tabular}

A mental health problem was diagnosed in 249 adolescents (6.4\%), 158 females (63.4\%). Table 1 also shows the primary symptoms reported by adolescents with these issues and the list of diagnoses related to mental health problems.

Excluding the traumatic causes of access, PED visits among adolescents were 2131, 249 (12\%) of whom reported a diagnosis of a mental health problem.

Table 2 shows the number of urgent presentations and different outcomes of adolescents compared younger patients presenting to the PED. Notably, despite a similar rate of hospitalization, the adolescents were admitted more frequently to the neuropsychiatric ward $(p<0.001)$. 
Table 2

Outcomes of adolescents compared to children accessing to the PED

\begin{tabular}{|c|c|c|c|}
\hline & $\begin{array}{l}\text { Accesses of adolescent } \\
(13-17 \mathrm{yrs})\end{array}$ & $\begin{array}{l}\text { Accesses of children } \\
(0-12 \mathrm{yrs})\end{array}$ & $\begin{array}{l}\mathrm{p}- \\
\text { value }\end{array}$ \\
\hline Patients, n (\%) & 4539 (18.5) & $20060(81.5)$ & - \\
\hline $\begin{array}{l}\text { Age in years, mean, standard } \\
\text { deviation }\end{array}$ & $15+/-1.8$ & $6+/-3.7$ & - \\
\hline Female, n (\%) & $1310(42.8)$ & $9513(47.4)$ & - \\
\hline Triage code, $\mathrm{n}(\%)$ & $840(18.5)$ & $6563(32.7)$ & \multirow{2}{*}{$<001$} \\
\hline - White (not urgent) & $3183(70.1)$ & $12890(64.3)$ & \\
\hline - Green (minor urgencies) & $511(11.2)$ & $1207(6.0)$ & 0.001 \\
\hline \multirow{2}{*}{$\begin{array}{l}\text { - Yellow (urgent) } \\
\text { - Red (emergent/resuscitation) }\end{array}$} & \multirow[t]{2}{*}{$5(0.1)$} & \multirow[t]{2}{*}{$44(0.2)$} & \multirow{2}{*}{$\begin{array}{l}< \\
0.001 \\
0.14\end{array}$} \\
\hline & & & \\
\hline Outcome, n (\%) & $4397(96.4)$ & $19514(97.2)$ & 0.13 \\
\hline - Discharge & $142(3.6)$ & $546(2.8)$ & \\
\hline \multicolumn{4}{|l|}{ - Hospitalization } \\
\hline \multirow{2}{*}{$\begin{array}{l}\text { Wards of destination in hospitalized } \\
\text { patients, } n(\%)\end{array}$} & $54(38)$ & $22(4.0)$ & \multirow{2}{*}{$<.001$} \\
\hline & $35(24.6)$ & 202 (36.9) & \\
\hline \multirow{2}{*}{ - Pediatric } & $24(17)$ & 87 (15.9) & 0.0 .47 \\
\hline & $14(9.8)$ & $101(18.5)$ & 0.81 \\
\hline - Orthopedic & $15(11.2)$ & $134(24.5)$ & 0.03 \\
\hline - Surgery & & & 0.02 \\
\hline - Others & & & \\
\hline
\end{tabular}

\section{Discussion}

This study showed that mental health problems are a leading cause of urgent hospitalization in adolescence. In this series, adolescent patients accounted for nearly a quarter of patients who accessed the PED, and this percentage was in line with that of a previous study. (7)

The principal cause of adolescents' access to PED was trauma, but the presentation of mental health problems was considerably high: $6.4 \%$ of adolescents received a diagnosis related to mental health, with anxiety and behavioral disorders as the most common diagnoses. Comparing our results with those of a previous study, performed in a similar setting in 1990's, a rise in the prevalence of visits for mental health problems is evident, $6.4 \%$ vs. $1.6 \%$, respectively. (11) We found that adolescent girls were more prone to 
present with a mental health problem than male peers, and this data was in line with previous studies. (6, $11,12)$

Aside from traumas, among adolescents, 1 in 8 visits was related to a mental health problem, and a third of the hospitalized adolescents were referred to the neuropsychiatric ward. Admission to this ward was significantly more frequent in adolescents than in younger patients accessing the PED $(p<0.001)$.

Evidence shows that near $20 \%$ of adolescents present with a mental health problem. (13) Patients with these diseases often seek help, with an increasing trend in recent years, in the emergency services, (1418) which for most of them are the first contact with the health care system. Therefore, pediatricians working in the emergency room should be prepared to receive and perform an appropriate investigation in these patients. In order to better evaluate these subjects, specific screening tools have been developed, (16) recommending the need for routine assessment of the family and social functioning, substance abuse, emotional and behavioral distress, with particular attention to suicidal thoughts. We want to emphasize that, in our series, nearly $25 \%$ of adolescents diagnosed with a mental health problem, presented with physical symptoms such as headache, abdominal pain, chest pain, respiratory distress, or dizziness. Thus, confirming that in adolescence, mental health problems frequently hide behind physical complaints. Besides, in just one year, six adolescents presented for a suicide attempt, confirming the high risk of suicide and self-harm in this age group. (19)

The few available studies performed in a PED setting focused on adolescents showing that the main reasons for the PED access were injuries or exacerbations of chronic diseases such as asthma or diabetes. $(7,8)$ On the contrary, this study revealed the significant burden of mental health problems in patients' populations, further raising awareness of these disorders in adolescence. In our setting, these diseases are the first cause of urgent hospitalization in this age group. These data can contribute to highlight the need for an implementation of specially equipped staff and wards to support these patients, across Institutions.

Our study has some limitations. It was a single-center study, referring to a tertiary-level children's hospital in a medium-sized city, so our results cannot be generalized; however, the substantial number of visits recorded strengthened our findings. Our Institution doesn't have a psychiatric emergency clinic, but we highlight that it has a neuropsychiatric ward. In Italy, many PED don't have the possibility to admit patients with mental health problems to a neuropsychiatric ward because this specific ward is absent in many Institutions. This was a retrospective study, so we cannot rule out misclassification of patients, and, finally, patients who access our PED are aged 0 to 17 years old, therefore, we cannot provide data for late adolescence, 18 to 25 years of age.

In conclusion, this study described the features of a cohort of adolescents accessing the PED for a whole year. It highlighted the remarkable burden of mental health problems in this cohort of patients, showing that it is the first cause of urgent hospitalization in this age group. 


\section{Abbreviations}

PED

pediatric emergency department

\section{Declarations}

\section{Acknowledgments}

The authors thank Martina Bradaschia for the English revision of the manuscript.

\section{Authors' contributions}

GC and EB conceived the study. GC, LP, CG designed the study protocol. LP, RG, IR contributed to the acquisition of the data. MG contributed to the statistical analysis of the data and reviewed the final version of the paper. GC, AA, LB and EB wrote down the draft of the paper and contributed to the critical revision of the work. All the authors read and approved the final manuscript.

\section{Funding}

The authors did not receive any funding or grant for this work.

\section{Availability of data and material}

The datasets used and/or analysed during the current study available from the Clinical Epidemiology and Public Health Research Unit of the IRCCS Burlo Garofolo, Trieste, Italy.

\section{Ethics approval and consent to participate}

The study was approved by the Institutional Review Board of the Institute for Maternal and Child Health IRCCS Burlo Garofolo, Trieste, Italy.

\section{Consent for publication}

Not applicable

\section{Competing interests}

The authors declare that they have no competing interests.

\section{References}

1. Patton GC, Sawyer SM, Santelli JS, Ross DA, Afifi R, Allen NB, et al. Our future: a Lancet commission on adolescent health and wellbeing. Lancet. 2016;387:2423-2478. 
2. Cousijn J, Luiten M, Feldstein Ewing SW. Adolescent resilience to addiction: a social plasticity hypothesis. Lancet Child Adolesc Health. 2018;2:69-78.

3. Ferrari A, Bleyer A. Participation of adolescents with cancers in clinical trial. Cancer Treat Rev. 2007;33:603-8.

4. Santelli JS, Sivaramakrishnan K, Edelstein Zr, Dried LP. Adolescent Risk-Taking, Cancer Risk, and Life Course Approaches to Prevention. J Adolesc Health. 2013;52:S41-4.

5. Barr RD, Ferrari A, Ries L, Whelan J, Bleyer A. Cancer in Adolescents and Young Adults A Narrative Review of the Current Status and a View of the Future. JAMA Pediatr. 2016;170:495-501.

6. Gunnell D, Kidger J, Elvidge H. Adolescent mental health in crisis. BMJ. 2018;k2608.

7. Melzer-Lange M, Lye PS. Adolescent health care in a pediatrics emergency department. Ann Emerg Med. 1996;27:633-7.

8. Marsh RH, Mollen CJ, Shofer FS and Baren JM. Characteristics that distinguish adolescents who present to a children's hospital emergency department from those presenting to a general emergency department. Pediatr Emerg Care. 2009;25:376-9.

9. Rasquin A, Di Lorenzo C, Forbes D, Guiraldes E, Hyams JS, Staiano A, et al. Childhood functional gastrointestinal disorders: child/adolescent. Gastroenterology. 2006;130:1527-1537.

10. Headache Classification Committee International Headache Society. The International Classification of Headache Disorders, 3rd edition (beta version). 2013;33:629-808.

11. Sills MR,Bland SD. Summary statistics for pediatric psychiatric visits to US emergency departments, 1993-1999. Pediatrics. 2002;110:e40.

12. Collishaw S, Hammerton G, Mahedy L, Sellers R, Owen MJ, Craddock N, et al. Mental health resilience in the adolescent off spring of parents with depression: a prospective longitudinal study. Lancet Psychiatry. 2016;3:49-57.

13. Chun TH, Mace SE, Katz ER. American Academy of Pediatrics; Committee on Pediatric Emergency Medicine, and American College of Emergency Physicians; Pediatric Emergency Medicine Committee. Pediatrics. 2016;138:e20161570.

14. Sheridan DC, Spiro DM, Fu R, Johnson KP, Sheridan JS, Oue AA, et al. Mental Health Utilization in a Pediatric Emergency Department. Pediatr Emerg Care. 2015;31:555-9.

15. Cozzi G, Minute M, Skabar A, Pirrone A, Jaber M, Neri E, Montico M, et al. Somatic symptom disorder was common in children and adolescents attending an emergency department complaining of pain. Acta Paediatr. 2017;106:586-593.

16. Cappelli M, Gray C, Zemek R, Cloutier P, Kennedy A, Glennie E, et al. The HEADS-ED: a rapid mental health screening tool for pediatric patients in the emergency department. 2012;130:e321-7.

17. Cozzi G, Minute M, Ventura G, Barbi E. Mental Health Problems in Children and Adolescents in the Emergency Department: "The Times They Are A-Changin"'. Pediatr Emerg Care. 2017;33:e8.

18. Gill PJ, Saunders N, Gandhi S, Gonzalez A, Kurdyak P, Vigod S, Guttmann A. Emergency Department as a First Contact for Mental Health Problems in Children and Youth. J Am 
Acad Child Adolesc Psychiatry. 2017;56:475-482.e4.

19. Zanus C, Battistutta S, Aliverti R, Montico M, Cremaschi S, Ronfani L, Monasta L, Carrozzi M. Adolescent Admissions to Emergency Departments for Self-Injurious Thoughts and Behaviors. PLoS One. 2017;12:e0170979. 